\title{
REALIZATION OF 2D (2,2)-PERIODIC ENCODERS BY MEANS OF 2D PERIODIC SEPARABLE ROESSER MODELS
}

\author{
Diego NAPP ${ }^{a}, \operatorname{RiCARDO}_{\text {PEREIRA }}{ }^{a, *}, \mathrm{RAQUEL}_{\mathrm{PINTO}}{ }^{a}, \mathrm{PAUlA}_{\mathrm{ROCHA}}{ }^{b}$ \\ ${ }^{a}$ CIDMA, Department of Mathematics \\ University of Aveiro, Campus Universitário de Santiago, 3810-193 Aveiro, Portugal \\ e-mail: \{diego, ricardopereira, raquel\}@ua.pt \\ ${ }^{b}$ SYSTEC, Faculty of Engineering \\ University of Porto, R. Dr. Roberto Frias, 4200-465 Porto, Portugal \\ e-mail: mprocha@fe.up.pt
}

\begin{abstract}
It is well known that convolutional codes are linear systems when they are defined over a finite field. A fundamental issue in the implementation of convolutional codes is to obtain a minimal state representation of the code. Compared with the literature on one-dimensional (1D) time-invariant convolutional codes, there exist relatively few results on the realization problem for time-varying 1D convolutional codes and even fewer if the convolutional codes are two-dimensional (2D). In this paper we consider 2D periodic convolutional codes and address the minimal state space realization problem for this class of codes. This is, in general, a highly nontrivial problem. Here, we focus on separable Roesser models and show that in this case it is possible to derive, under weak conditions, concrete formulas for obtaining a 2D Roesser state space representation. Moreover, we study minimality and present necessary conditions for these representations to be minimal. Our results immediately lead to constructive algorithms to build these representations.
\end{abstract}

Keywords: periodic 2D systems, convolutional codes, realizations.

\section{Introduction}

Since the 1960 s it has been widely known that convolutional codes and linear systems which are defined over a finite field are mathematically identical (Rosenthal, 2001). In the last decades there has been a new and increased interest in this connection, and many advances have been made using the system-theoretical framework when dealing with convolutional codes. This approach has led to significant progress in fundamental issues in the area (Gluesing-Luerssen and Schneider, 2007; Rosenthal and York, 1999; Rosenthal, 2001; Kuijper and Polderman, 2004).

Multi-dimensional convolutional codes $(n \mathrm{D}$ convolutional codes, where $n$ stands for the dimension) are a natural generalization of one-dimensional (1D) convolutional codes. Standard 1D convolutional codes deal with the transmission and storage of data that evolve over time. Instead, $n \mathrm{D}$ convolutional codes are suited for dealing with $n$ dimensional data, e.g., pictures, storage

* Corresponding author media, etc. (see Basu and Swamy, 2002). However, while the 1D convolutional codes have been thoroughly understood, little research has been done in the area of $n \mathrm{D}$ convolutional codes and much more needs to be done to make it attractive for applications.

The literature on $n \mathrm{D}$ convolutional codes is limited, but some important fundamental results have already been obtained. The algebraic theory of $2 \mathrm{D}$ and $n \mathrm{D}$ convolutional codes has been laid out by Valcher and Fornasini (1994), Fornasini and Valcher (1998; 1994), Gluesing-Luersen et al. (2000), Lobo et al. (2012) and Weiner (1998); see also the references therein. They introduced the general theory for the study of $n \mathrm{D}$ convolutional codes constituted by sequences indexed on $\mathbb{Z}^{n}$ or $\mathbb{N}^{n}$, and discussed issues such as the characterization of such codes in terms of their internal properties and input-output representations.

A fundamental issue that arises in this context is the so-called minimal realization problem: How to derive a state-space representation of the code with the 
minimal dimension (properly defined below) (see Napp et al., 2010; Fornasini and Pinto, 2004; Jangisarakul and Charoenlarpnopparut, 2011; Charoenlarpnopparut and Bose, 2001). This representation is essential as it represents a blueprint for an actual physical device, typically built from shift registers. These representations are also of paramount importance for deriving efficient decoding algorithms using trellis diagrams, e.g., the Viterbi decoding algorithm.

The minimal state space realization problem plays a particularly important role in the analysis and design of multi-dimensional convolutional codes because of the large amount of data involved. However, the general problem of a minimal state space realization of multidimensional systems has not been solved even for 2-dimensional systems. Nevertheless, for some special cases, minimal state space realization methods have been derived; see the works of Zerz (2000), Pinho et al. (2014), Pinho (2016), Napp et al. (2010), Galkowski (1996; 2001) and the references therein.

The state space formulation of convolutional codes can easily be extended to the time-varying case. The system matrices describing the convolutional code are typically regarded to be constant over time. However, one can also consider time-varying linear systems in which the matrices representing the code also depend on time. The idea of considering 1D time-varying and, in particular, periodically time-varying convolutional codes has attracted considerable attention of many researchers. After Costello (1974) conjectured that time-varying convolutional codes can achieve better properties than time-invariant ones, many researchers have investigated such codes. The research in this area has focused on finding concrete encoders that yield 1D periodic convolutional codes with good distance properties (Mooser, 1983; Palazzo, 1993; Felstrom and Zigangirov, 1999; Guardia, 2019) and on state space representations of time-varying systems (Napp et al., 2019; Climent et al., 2009; Aleixo et al., 2011; Kuijper and Willems, 1997).

In this paper we continue this thread of research by considering periodic $2 \mathrm{D}$ convolutional codes and the corresponding minimal state space realization problem. Although some results have been obtained in the context of time-invariant 2D convolutional codes (Pinho et al., 2014; Fornasini et al., 2015) and 1D periodic convolutional codes (Climent et al., 2009), this problem remains unexplored in the context of periodic $2 \mathrm{D}$ convolutional codes. Here we aim at deriving state Roesser 2D state space representations (Aleixo and Rocha, 2017; Kaczorek, 2001) from a (2,2)-periodic two-dimensional generator matrix. This is, in general, a highly nontrivial problem and one needs to assume additional conditions to be able to build minimal state space representations. In this work we study the case of separable (2,2)-periodic two-dimensional generator matrices, i.e., the encoders $G\left(z_{1}, z_{2}\right)$ that can be decomposed as $G\left(z_{1}, z_{2}\right)=V\left(z_{2}\right) H\left(z_{1}\right)$, where $V\left(z_{2}\right)$ and $H\left(z_{1}\right)$ are polynomial matrices with periodically time-varying coefficients of period 2. Specifically, both $V\left(z_{2}\right)$ and $H\left(z_{1}\right)$ are constructed based on two alternating invariant encoders $V_{0}\left(z_{2}\right)$ and $V_{1}\left(z_{2}\right)$, and $H_{0}\left(z_{1}\right)$ and $H_{1}\left(z_{1}\right)$, respectively. We first show that one cannot expect to obtain a realization of the periodic 2D convolutional code by realizing independently the time-invariant encoders on which $V\left(z_{2}\right)$ and $H\left(z_{1}\right)$ are based. However, we provide certain conditions that allow obtaining a minimal state Roesser 2D state space representation. Moreover, our results are constructive in the sense that we provide explicit formulas for the realization and a concrete methodology for obtaining such representations.

\section{Prelimiaries}

2.1. Time-invariant convolutional codes. Let $\mathbb{F}$ be a finite field and $\mathbb{F}[z]$ the polynomial ring. In a module theoretic point of view, we define a convolutional code as follows.

Definition 1. Let $\mathbb{F}$ be a finite field and $n, k$ positive integers with $k<n$. A time-invariant convolutional code $\mathcal{C}$ of rate $k / n$ is a submodule $\mathbb{F}^{n}[z]$ described as

$$
\mathcal{C}=\left\{w(z) \in \mathbb{F}^{n}[z]: w(z)=G(z) u(z), u(z) \in \mathbb{F}^{k}[z]\right\},
$$

where $G(z) \in \mathbb{F}^{n \times k}[z]$ is a full column rank $n \times k$ polynomial matrix over $\mathbb{F}$, called the encoder, $u(z)$ taking values in $\mathbb{F}^{k}[z]$ is the information vector and $w(z)$ is the codeword.

The encoders of a code $\mathcal{C}$ are not unique; however, they only differ by right multiplication by unimodular matrices over $\mathbb{F}[z]$. An encoder $G(z)$ is called column reduced if the sum of its column degrees attains the minimal possible value among all the encoders of the same code. If $G(z) \in \mathbb{F}^{n \times k}[z]$ has column degrees $\nu_{1}, \ldots, \nu_{k}$, it can be written as

$$
G(z)=G_{\mathrm{hc}}\left[\begin{array}{llll}
z^{\nu_{1}} & & & \\
& z^{\nu_{2}} & & \\
& & \ddots & \\
& & & z^{\nu_{k}}
\end{array}\right]+G_{\mathrm{rem}}(z)
$$

where $G_{\text {rem }}(z)$ is a polynomial matrix such that the degree of column $i$ is less than $\nu_{i}, i=1, \ldots, k$, and $G_{\mathrm{hc}} \in \mathbb{F}^{n \times k}$ is a matrix whose $i$-th column contains the coefficients of $z^{\nu_{i}}$ in the $i$-th column of $G(z) . G_{\mathrm{hc}}$ is called the leading column coefficient matrix and $G(z)$ is column reduced if and only if $G_{\mathrm{hc}}$ is full column rank.

We define the degree $\delta$ of a convolutional code as the sum of the column degrees of one (and hence any) column reduced encoder. Note that the list of column degrees (also known as Forney indices) of a column reduced encoder is 
unique up to a permutation. A code $\mathcal{C}$ of rate $k / n$ and degree $\delta$ is said to be an $(n, k, \delta)$ code.

2.2. Periodically time-varying $1 \mathrm{D}$ convolutional codes. In this section we consider 1D convolutional codes $\mathcal{C}$ with 2-periodic encoders. The definition of such encoders (or encoding maps) is introduced next, together with the definition of the corresponding 2-periodic (time-varying) convolutional codes see (Costello, 1974; Palazzo, 1993).

Definition 2. Given two polynomial matrices $G^{0}(z)$, $G^{1}(z) \in \mathbb{F}^{n \times k}[z]$, the periodic encoding map induced by $G^{0}$ and $G^{1}$ is defined as

$$
\Phi_{G^{0}, G^{1}}:\left\{\begin{array}{l}
\mathbb{F}^{k}[z] \longrightarrow \mathbb{F}^{n}[z], \\
u(z) \longmapsto w(z),
\end{array}\right.
$$

where $w(z)=\sum_{i=0}^{+\infty} w_{i} z^{i}$ with the abbreviated notation $w_{2 \ell+t}=\left(G^{t}(z) u(z)\right)_{2 \ell+t}, t=0,1, \ell \in \mathbb{N}_{0}$. Here $\left(G^{t}(z) u(z)\right)_{2 \ell+t}$ represents the $(2 \ell+t)$-coefficient of the polynomial $G^{t}(z) u(z)$.

The corresponding periodic convolutional code $\mathcal{C}_{p}$ is

$$
\begin{array}{r}
\mathcal{C}_{p}=\left\{w(z) \in \mathbb{F}^{n}[z]: w(z)=\Phi_{G^{0}, G^{1}}(u(z)),\right. \\
\left.u(z) \in \mathbb{F}^{k}[z]\right\} .
\end{array}
$$

Such codes will be called 2-periodic convolutional codes.

2.3. State-space realizations. In systems theory, input-state-output models are mainly used to describe the time evolution of the system signals, which, in the discrete-time case, are time sequences. Therefore, in the sequel, we sometimes identify an element $a(z)=$ $\sum_{i=0}^{N} a_{i} z^{i} \in \mathbb{F}[z]$ with the finite support sequence $a_{0}=$ $(a(z))_{0}, a_{1}=(a(z))_{1}, \ldots, a_{N}=(a(z))_{N}$ formed by its coefficients, and also use the notation $a(\ell)$ to write $a_{\ell}=$ $(a(z))_{\ell}$. The same applies to vectors with components in $\mathbb{F}[z]$.

A state-space system

$$
\left\{\begin{aligned}
x(\ell+1) & =A x(\ell)+B u(\ell), \\
w(\ell) & =C x(\ell)+D u(\ell),
\end{aligned}\right.
$$

$\ell \in \mathbb{N}_{0}$, denoted by $(A, B, C, D)$, where $A \in \mathbb{F}^{\delta \times \delta}, B \in$ $\mathbb{F}^{\delta \times k}, C \in \mathbb{F}^{n \times \delta}$ and $D \in \mathbb{F}^{n \times k}$, is said to be a state-space realization of the time-invariant $(n, k, \delta)$ convolutional code $\mathcal{C}$ if $\mathcal{C}$ is the set of codewords $w(z) \in$ $\mathbb{F}^{n}[z]$ identified with the finite support output sequences $w$ corresponding to finite support input sequences $u$ (i.e., to information sequences $\left.u(z) \in \mathbb{F}^{k}[z]\right)$ and zero initial conditions, i.e., $x(0)=0$.

If $G(z) \in \mathbb{F}^{n \times k}[z]$ is an encoder of $\mathcal{C},(A, B, C, D)$ is a state-space realization of $G(z)$ if

$$
G(z)=C(I-A z)^{-1} B z+D .
$$

If $G(z)=\sum_{i \in \mathbb{N}} G_{i} z^{i}$, with $G_{i} \in \mathbb{F}^{n \times k}$, then

$$
G_{0}=D, \quad G_{i}=C A^{i-1} B, \quad i \geq 1 .
$$

Note that $G(z)$ admits many realizations. It is well known that a state-space realization $(A, B, C, D)$ of $G(z)$ is minimal, i.e., has a minimal dimension among all the realizations of $G(z)$, if $(A, B)$ is controllable and $(A, C)$ is observable, i.e., the polynomial matrices $\left[z^{-1} I-A \mid B\right]$ and $\left[z^{-1} I-A\right]$ have, respectively, right and left polynomial inverses (in $z^{-1}$ ). The minimal dimension of a state-space realization of $G(z)$ is called the McMillan degree (Kailath, 1980) of $G(z)$ and it is represented as $\mu(G)$.

The next proposition, adapted from the works of Fornasini and Pinto (2004) or Gluesing-Luerssen and Schneider (2007), provides a state-space realization for a given (not necessarily column reduced) encoder. Moreover, it states that state-space realizations of a code can be obtained from minimal realizations of column reduced encoders.

Proposition 1. Let $G(z) \in \mathbb{F}^{n \times k}[z]$ be a polynomial matrix with rank $k$ and column degrees $\nu_{1}, \ldots, \nu_{k}$. Consider $\bar{\delta}=\sum_{i=1}^{k} \nu_{i}$. Let $G(z)$ have columns $g_{i}(z)=$ $\sum_{\ell=0}^{\nu_{i}} g_{\ell, i} z^{\ell}, i=1, \ldots, k$ where $g_{\ell, i} \in \mathbb{F}^{n}$. For $i=$ $1, \ldots, k$ define the matrices

$$
\begin{gathered}
A_{i}=\left[\begin{array}{cccc}
0 & \cdots & \cdots & 0 \\
1 & & & \vdots \\
& \ddots & & \vdots \\
& & 1 & 0
\end{array}\right] \in \mathbb{F}^{\nu_{i} \times \nu_{i}}, \quad B_{i}=\left[\begin{array}{c}
1 \\
0 \\
\vdots \\
0
\end{array}\right] \in \mathbb{F}^{\nu_{i}}, \\
C_{i}=\left[\begin{array}{lll}
g_{1, i} & \cdots & g_{\nu_{i}, i}
\end{array}\right] \in \mathbb{F}^{n \times \nu_{i}} .
\end{gathered}
$$

Then a state-space realization of $G$ is given by the matrix quadruple $(A, B, C, D) \in \mathbb{F}^{\bar{\delta} \times \bar{\delta}} \times \mathbb{F}^{\bar{\delta} \times k} \times \mathbb{F}^{n \times \bar{\delta}} \times \mathbb{F}^{n \times k}$, where

$$
\begin{aligned}
A & =\left[\begin{array}{lll}
A_{1} & & \\
& \ddots & \\
& & A_{k}
\end{array}\right], \\
B & =\left[\begin{array}{lll}
B_{1} & & \\
& \ddots & B_{k}
\end{array}\right], \\
C & =\left[\begin{array}{lll}
C_{1} & \cdots & C_{k}
\end{array}\right], \\
D & =\left[\begin{array}{lll}
g_{0,1} & \cdots & g_{0, k}
\end{array}\right]=G(0) .
\end{aligned}
$$

In the case where $\nu_{i}=0$, the $i$-th blocks of $A$ and $C$ are void and in $B$ a zero column occurs.

In this realization, $(A, B)$ is controllable and, if $G(z)$ is a column reduced encoder, $(A, C)$ is observable and therefore the realization of $G(z)$ is minimal. Thus, the McMillan degree of a column reduced encoder is equal to the sum of its column degrees. 
2.4. State-space realizations of $1 D$ periodic convolutional codes.

Definition 3. Let $\Sigma_{i}=\left(A_{i}, B_{i}, C_{i}, D_{i}\right), i=0,1$, be two state-space systems with the same dimension. We define a periodic state-space system $\Sigma_{p}$ as

$$
\left\{\begin{aligned}
x(\ell+1) & =A(\ell) x(\ell)+B(\ell) u(\ell), \\
w(\ell) & =C(\ell) x(\ell)+D(\ell) u(\ell),
\end{aligned}\right.
$$

$\ell \in \mathbb{N}_{0}$, where $A(\cdot), B(\cdot), C(\cdot), D(\cdot)$ are periodic functions with period 2 , such that

$$
(A(2 j), B(2 j), C(2 j), D(2 j))=\left(A_{0}, B_{0}, C_{0}, D_{0}\right)
$$

and

$$
\begin{gathered}
(A(2 j+1), B(2 j+1), C(2 j+1), D(2 j+1)) \\
=\left(A_{1}, B_{1}, C_{1}, D_{1}\right), \quad j \in \mathbb{N}_{0} .
\end{gathered}
$$

The dimension of $\Sigma_{p}$ is defined as that of the state vector $x$. In this case we say that $\Sigma_{p}$ is obtained from $\Sigma_{0}$ and $\Sigma_{1}$.

Moreover, $\Sigma_{p}$ is a realization of a periodic encoding map $\Phi_{G^{0}, G^{1}}$ if the output of $\Sigma_{p}$ that corresponds to an input $u(z)$ is equal to $\Phi_{G^{0}, G^{1}}(u(z))$, for all $u(z) \in \mathbb{F}^{k}[z]$.

Let $\Sigma_{0}$ and $\Sigma_{1}$ be two state-space realizations (of the same dimension) of two encoders $G^{0}(z)$ and $G^{1}(z)$. It is possible to show that the 2 -periodic system $\Sigma_{p}$ obtained from $\Sigma_{0}$ and $\Sigma_{1}$ is not always a state-space realization of $\Phi_{G^{0}, G^{1}}$

However, in the next theorem (Napp et al., 2019) we provide a sufficient condition for a periodic state-space system to be a realization of a periodic encoding map.

Theorem 1. Consider two encoders $G^{0}(z) \in \mathbb{F}^{n \times k}[z]$ and $G^{1}(z) \in \mathbb{F}^{n \times k}[z]$ with the same column degrees, and let $\Sigma_{i}$ be the realizations of $G^{i}(z), i=0,1$, obtained by Proposition 1] Then the periodic state-space system $\Sigma_{p}$ obtained from $\Sigma_{0}$ and $\Sigma_{1}$ is a realization of the periodic encoding map $\Phi_{G^{0}, G^{1}}$.

When the encoders given in the previous theorem are column reduced, then the realization of the corresponding encoding map is minimal, as stated next (Napp et al., 2019).

Theorem 2. Let $G^{0}(z), G^{1}(z) \in \mathbb{F}^{n \times k}[z]$ be two column reduced encoders with the same column degrees, and let $\Sigma_{i}$ be the realizations of $G^{i}(z), i=0,1$, obtained by Proposition 11 Then the 2-periodic state-space realization of the periodic encoding map $\Phi_{G^{0}, G^{1}}$ obtained from $\Sigma_{0}$ and $\Sigma_{1}$ is minimal.

\section{2D (2,2)-periodic convolutional codes}

In this paper we consider $2 \mathrm{D}$ convolutional codes $\mathcal{C}$ with $(2,2)$-periodic encoders. Analogously to the 1D case, we introduce the definition of a periodic encoding map.
Definition 4. Given four 2D polynomial matrices $G^{00}\left(z_{1}, z_{2}\right), G^{10}\left(z_{1}, z_{2}\right), G^{01}\left(z_{1}, z_{2}\right), G^{11}\left(z_{1}, z_{2}\right) \in$ $\mathbb{F}^{n \times k}\left[z_{1}, z_{2}\right]$, the (2,2)-periodic encoding map induced by $G^{00}, G^{10}, G^{01}$ and $G^{11}$ is defined as

$$
\Phi_{G^{00}, G^{10}, G^{01}, G^{11}}:\left\{\begin{array}{l}
\mathbb{F}^{k}\left[z_{1}, z_{2}\right] \longrightarrow \mathbb{F}^{n}\left[z_{1}, z_{2}\right], \\
u\left(z_{1}, z_{2}\right) \longmapsto\left(z_{1}, z_{2}\right),
\end{array}\right.
$$

where $w\left(z_{1}, z_{2}\right)=\sum_{(i, j) \in \mathbb{N}^{2}} w_{i, j} z_{1}^{i} z_{2}^{j}$ and

$$
\begin{array}{r}
w_{2 \ell+i, 2 m+j}=\left(G^{i j}\left(z_{1}, z_{2}\right) u\left(z_{1}, z_{2}\right)\right)_{2 \ell+i, 2 m+j}, \\
i, j=0,1, \quad \ell, m \in \mathbb{N}_{0},
\end{array}
$$

the quantity $\left(G^{i j}\left(z_{1}, z_{2}\right) u\left(z_{1}, z_{2}\right)\right)_{2 \ell+i, 2 m+j}$ represents the $(2 \ell+i, 2 m+j)$-coefficient of the polynomial $G^{i j}\left(z_{1}, z_{2}\right) u\left(z_{1}, z_{2}\right)$.

The corresponding $2 D(2,2)$-periodic convolutional code $\mathcal{C}_{p}$ is

$$
\begin{aligned}
\mathcal{C}_{p}=\{ & w\left(z_{1}, z_{2}\right) \in \mathbb{F}^{n}\left[z_{1}, z_{2}\right]: \\
& \left.\exists u\left(z_{1}, z_{2}\right) \in \mathbb{F}^{k}\left[z_{1}, z_{2}\right] \text { such that (4) holds }\right\}, \\
& w\left(z_{1}, z_{2}\right)=\Phi_{G^{00}, G^{10}, G^{01}, G^{11}}\left(u\left(z_{1}, z_{2}\right)\right) .
\end{aligned}
$$

Such codes will be called $2 D(2,2)$-periodic convolutional codes.

We consider a special class of 2D polynomial matrices $G^{i j}\left(z_{1}, z_{2}\right)$ that can be factored as

$$
G^{i j}\left(z_{1}, z_{2}\right)=V^{j}\left(z_{2}\right) H^{i}\left(z_{1}\right),
$$

where $H^{i}\left(z_{1}\right) \in \mathbb{F}^{q \times k}\left[z_{1}\right]$ and $V^{j}\left(z_{2}\right) \in \mathbb{F}^{n \times q}\left[z_{2}\right]$ are 1D polynomial matrices, $i=0,1$.

Therefore, the previous 2D $(2,2)$-periodic convolutional code $\mathcal{C}_{p}$ can be defined as

$$
\begin{aligned}
& \mathcal{C}_{p}=\left\{w\left(z_{1}, z_{2}\right) \in \mathbb{F}^{n}\left[z_{1}, z_{2}\right]:\right. \\
&\left.\quad \exists u\left(z_{1}, z_{2}\right) \in \mathbb{F}^{k}\left[z_{1}, z_{2}\right] \text { such that } 5 \text { holds }\right\} \\
& w\left(z_{1}, z_{2}\right)=\Phi_{V^{0} H^{0}, V^{0} H^{1}, V^{1} H^{0}, V^{1} H^{1}}\left(u\left(z_{1}, z_{2}\right)\right)
\end{aligned}
$$

\section{2D state space realizations}

Here we focus on the state space realizations of the special class of $2 \mathrm{D}$ periodic convolutional codes introduced in the previous section by means of $2 \mathrm{D}$ periodic Roesser models. In general, this is a nontrivial matter, mainly due to the fact that a $2 \mathrm{D}$ periodic state space realization cannot be obtained by independently realizing each of the invariant polynomial operators $G^{i j}=V^{j} H^{i}$ (Aleixo and Rocha, 2017). However, in this paper we show that under certain conditions this problem does not arise, i.e., combining independent realizations of the invariant operators $G^{i j}$ does yield a 2D periodic realization of the corresponding 2D periodic convolutional code. Before 
presenting our result, we first consider the invariant 2D case.

As in Section 2.3, we sometimes identify an element

$$
a\left(z_{1}, z_{2}\right)=\sum_{i=0}^{N_{1}} \sum_{j=0}^{N_{2}} a_{i, j} z_{1}^{i} z_{2}^{j} \in \mathbb{F}\left[z_{1}, z_{2}\right]
$$

with the finite support sequence $a_{0,0}=\left(a\left(z_{1}, z_{2}\right)\right)_{0,0}$, $a_{1,0}=\left(a\left(z_{1}, z_{2}\right)\right)_{1,0}, \ldots, a_{N_{1}, N_{2}}=\left(a\left(z_{1}, z_{2}\right)\right)_{N_{1}, N_{2}}$ formed by its coefficients, and also write $a(\ell, m)$ to denote $a_{\ell, m}=\left(a\left(z_{1}, z_{2}\right)\right)_{\ell, m}$. The same applies to vectors with components in $\mathbb{F}\left[z_{1}, z_{2}\right]$.

4.1. Invariant $2 \mathrm{D}$ case. $\mathrm{As}$ is well known, in the $2 \mathrm{D}$ invariant case, a separable Roesser model realization for a code

$$
\begin{aligned}
\mathcal{C}= & \left\{w\left(z_{1}, z_{2}\right) \in \mathbb{F}^{n}\left[z_{1}, z_{2}\right]:\right. \\
& \left.w\left(z_{1}, z_{2}\right)=G\left(z_{1}, z_{2}\right) u\left(z_{1}, z_{2}\right), u\left(z_{1}, z_{2}\right) \in \mathbb{F}^{k}\left[z_{1}, z_{2}\right]\right\},
\end{aligned}
$$

where

$$
G\left(z_{1}, z_{2}\right)=V\left(z_{2}\right) H\left(z_{1}\right),
$$

can be obtained as the series connection of the 1D state space realizations of $H$ and $V$. Indeed, if $\left(A^{h}, B^{h}, \tilde{C}^{h}, D^{h}\right)$ and $\left(A^{v}, \tilde{B}^{v}, C^{v}, D^{v}\right)$ are respectively state space realizations of $H\left(z_{1}\right)$ and $V\left(z_{2}\right)$, then the separable Roesser model $\Sigma=\left(A^{h}, A^{v}, A^{v h}, B^{h}, B^{v}, C^{h}, C^{v}, D\right)$ :

$$
\left\{\begin{aligned}
x^{h}(i+1, j) & =A^{h} x^{h}(i, j)+B^{h} u(i, j), \\
x^{v}(i, j+1) & =A^{v h} x^{h}(i, j)+A^{v} x^{v}(i, j)+B^{v} u(i, j), \\
w(i, j) & =C^{h} x^{h}(i, j)+C^{v} x^{v}(i, j)+D u(i, j),
\end{aligned}\right.
$$

with $A^{v h}=\tilde{B}^{v} \tilde{C}^{h}, B^{v}=\tilde{B}^{v} D^{h}, C^{h}=D^{v} \tilde{C}^{h}$ and $D=$ $D^{v} D^{h}$, is a realization of $\mathcal{C}$ in the sense that the codewords $w$ in $\mathcal{C}$ coincide with the outputs of (6) produced by the same input $u$ with zero initial conditions, i.e., $x^{h}(0, j)=$ 0 and $x^{v}(i, 0)=0, i, j \in \mathbb{N}_{0}$. Moreover, we assume that the dimensions of the horizontal and vertical states, $x^{h}(i, j)$ and $x^{v}(i, j)$, are $\delta_{h}$ and $\delta_{v}$, respectively.

In the sequel the minimality of separable Roesser models will be studied. We start with some preliminary definitions and results.

Definition 5. The horizontal and vertical controllability matrices of the separable Roesser model (6) are defined respectively as

$$
\begin{aligned}
\mathscr{C}_{h} & =\left[B^{h}\left|A^{h} B^{h}\right| \ldots \mid\left(A^{h}\right)^{\delta_{h}-1} B^{h}\right] \\
& \in \mathbb{F}^{\delta_{h} \times k \delta_{h}}, \\
\mathscr{C}_{v}= & {\left[B_{\delta_{h}}\left|A^{v} B_{\delta_{h}}\right| \cdots \mid\left(A^{v}\right)^{\delta_{v}-1} B_{\delta_{h}}\right] } \\
\in & \mathbb{F}^{\delta_{v} \times \delta_{v} k\left(\delta_{h}+1\right)},
\end{aligned}
$$

with $B_{\delta_{h}}=\left[B^{v} \mid A^{v h} \mathscr{C}_{h}\right] \in \mathbb{F}^{\delta_{v} \times k\left(\delta_{h}+1\right)}$.
Definition 6. The vertical and horizontal observability matrices of the separable Roesser model (6) are defined respectively as

$$
\begin{aligned}
& \mathscr{O}_{v} \\
& =\left[\left(C^{v}\right)^{\top}\left|\left(C^{v} A^{v}\right)^{\top}\right| \cdots \mid\left(C^{v}\left(A^{v}\right)^{\delta_{v}-1}\right)^{\top}\right]^{\top} \\
& \in \mathbb{F}^{n \delta_{v} \times \delta_{v}}, \\
& \mathscr{O}_{h} \\
& =\left[\left(C_{\delta_{v}}\right)^{\top}\left|\left(C_{\delta_{v}} A^{h}\right)^{\top}\right| \cdots \mid\left(C_{\delta_{v}}\left(A^{h}\right)^{\delta_{h}-1}\right)^{\top}\right]^{\top} \\
& \in \mathbb{F}^{\delta_{h} n\left(\delta_{v}+1\right) \times \delta_{h}},
\end{aligned}
$$

with

$$
C_{\delta_{v}}=\left[\begin{array}{c}
C^{h} \\
\mathscr{O}_{v} A^{v h}
\end{array}\right] \in \mathbb{F}^{n\left(\delta_{v}+1\right) \times \delta_{h}} .
$$

The following proposition is well known 1

\section{Proposition 2.}

1. The pair $\left(A^{h}, B^{h}\right)$ is controllable if and only if $\operatorname{rank} \mathscr{C}_{h}=\delta_{h}$.

2. The pair $\left(A^{v}, B_{\delta_{h}}\right)$ is controllable if and only if $\operatorname{rank} \mathscr{C}_{v}=\delta_{v}$.

3. The pair $\left(A^{v}, C^{v}\right)$ is observable if and only if $\operatorname{rank} \mathscr{O}_{v}=\delta_{v}$.

4. The pair $\left(A^{h}, C_{\delta_{v}}\right)$ is observable if and only if $\operatorname{rank} \mathscr{O}_{h}=\delta_{h}$.

For separable Roesser models, separable controllability and separable observability are defined as follows.

Definition 7. The 2D separable Roesser model (6) is said to be

1. separately locally controllable if $\left(A^{h}, B^{h}\right)$ and $\left(A^{v}, B_{\delta_{h}}\right)$ are controllable;

2. separately locally observable if $\left(A^{v}, C^{v}\right)$ and $\left(A^{h}, C_{\delta_{v}}\right)$ are observable.

Hinamoto (1980) presented a necessary and sufficient condition for the minimality of a separable Roesser model, which we state in the next result using the language of codes.

Theorem 3. Let $G\left(z_{1}, z_{2}\right) \in \mathbb{F}^{n \times k}\left[z_{1}, z_{2}\right]$ be an encoder of a convolutional code $\mathcal{C}$. Then the separable Roesser model $\Sigma=\left(A^{h}, A^{v}, A^{v h}, B^{h}, B^{v}, C^{h}, C^{v}, D\right)$ given by (6) is a minimal realization of the encoder $G\left(z_{1}, z_{2}\right)$ if and only if is separately locally controllable and separately locally observable.

\footnotetext{
${ }^{1}$ Note that previously (before stating Proposition 1 we gave an alternative definition of a controllable and observable pair.
} 
In the next theorem we provide a simpler characterization for the minimality of a separable Roesser model.

Theorem 4. Let $G\left(z_{1}, z_{2}\right) \in \mathbb{F}^{n \times k}\left[z_{1}, z_{2}\right]$ be an encoder of a convolutional code $\mathcal{C}$. Then the separable Roesser model $\Sigma=\left(A^{h}, A^{v}, A^{v h}, B^{h}, B^{v}, C^{h}, C^{v}, D\right)$ given by (6) is a minimal realization of the encoder $G\left(z_{1}, z_{2}\right)$ if and only if the following conditions hold:

1. $\left(A^{h}, B^{h}\right)$ and $\left(A^{v},\left[B^{v} \mid A^{v h}\right]\right)$ are controllable.

2. $\left(A^{v}, C^{v}\right)$ and $\left(A^{h},\left[\begin{array}{c}C^{h} \\ A^{v h}\end{array}\right]\right)$ are observable.

The next two auxiliary lemmas immediately prove the previous theorem.

Lemma 1. The 2D separable Roesser model (6) is separately locally controllable if and only if $\left(A^{h}, B^{h}\right)$ and $\left(A^{v},\left[B^{v} \mid A^{v h}\right]\right)$ are controllable.

Proof.

(Necessity) By definition of separately locally controllable, we have that the matrices $\mathscr{C}_{h}$ and $\mathscr{C}_{v}$ have full row rank. Defining the matrices

$$
\begin{gathered}
M=\left[B^{v} \mid A^{v h}\right], \quad \overline{\mathscr{C}_{h}}=\left[\begin{array}{c|c}
I_{\delta_{v}} & 0 \\
\hline 0 & \mathscr{C}_{h}
\end{array}\right], \\
\overline{\overline{\mathscr{C}_{h}}}=\left[\begin{array}{ccc}
\overline{\mathscr{C}_{h}} & & 0 \\
& \ddots & \\
0 & & \overline{\mathscr{C}_{h}}
\end{array}\right],
\end{gathered}
$$

we have that

$$
B_{\delta_{h}}=\left[B^{v} \mid A^{v h} \mathscr{C}_{h}\right]=M \overline{\mathscr{C}_{h}}
$$

and, in turn,

$\mathscr{C}_{v}=\left[B_{\delta_{h}}\left|A^{v} B_{\delta_{h}}\right| \cdots \mid\left(A^{v}\right)^{\delta_{v}-1} B_{\delta_{h}}\right]=\overline{\mathscr{C}_{v}} \overline{\overline{\mathscr{C}_{h}}}$,

with

$$
\overline{\mathscr{C}_{v}}=\left[M\left|A^{v} M\right| \cdots \mid\left(A^{v}\right)^{\delta_{v}-1} M\right] .
$$

Since $\mathscr{C}_{h}$ has full row rank, clearly both matrices $\overline{\mathscr{C}_{h}}$ and $\overline{\overline{\mathscr{C}_{h}}}$ also have full row rank. Moreover, by hypothesis, $\mathscr{C}_{v}$ has full row rank, which implies that $\overline{\mathscr{C}_{v}}$ must also have full row rank. This means that $\left(A^{v}, M\right)=\left(A^{v},\left[B^{v} \mid A^{v h}\right]\right)$ is controllable.

(Sufficiency) Assuming the hypothesis, it suffices to prove that the pair $\left(A^{v}, B_{\delta_{h}}\right)$ is controllable, i.e., that the matrix $\mathscr{C}_{v}$ has full row rank. Adopting the notation of the necessity part, we have that

$$
\mathscr{C}_{v}=\overline{\mathscr{C}_{v}} \overline{\overline{\mathscr{C}_{h}}} .
$$

Since $\left(A^{v},\left[B^{v} \mid A^{v h}\right]\right)$ is controllable, $\overline{\mathscr{C}_{v}}$ has full row rank. Furthermore, $\overline{\overline{\mathscr{C}_{h}}}$ has full row rank because $\mathscr{C}_{h}$ also does so by the hypothesis of controllability of $\left(A^{h}, B^{h}\right)$, and the result follows.

Lemma 2. The 2D separable Roesser model (6) is separately locally observable if and only if $\left(A^{v}, C^{v}\right)$ and $\left(A^{h},\left[\begin{array}{l}C^{h} \\ A^{v h}\end{array}\right]\right)$ are observable.

Proof. The proof is analogous to the one of the previous lemma.

4.2. Periodic 2D case. Analogously to the invariant $2 \mathrm{D}$ case, under certain conditions, in the $2 \mathrm{D}$ periodic case, a periodic separable Roesser model realization can be obtained as a series connection of two 1D periodic state space realizations of periodic operators $H^{2 k+i} \equiv H^{i}$ and $V^{2 \ell+j} \equiv V^{j}, i, j=0,1, k, l \in \mathbb{Z}$.

Consider the (2,2)-periodic encoding map

$$
\Phi_{V^{0} H^{0}, V^{0} H^{1}, V^{1} H^{0}, V^{1} H^{1}},
$$

and let further $\Sigma_{i}^{h}=\left(A_{i}^{h}, B_{i}^{h}, \tilde{C}_{i}^{h}, D_{i}^{h}\right)$ and $\Sigma_{j}^{v}=$ $\left(A_{j}^{v}, \tilde{B}_{j}^{v}, C_{j}^{v}, D_{j}^{v}\right)$ be state space realizations of the invariant operators $H^{i}$ and $V^{j}, i, j=0,1$, respectively. Assume that $\Sigma_{0}^{h}$ and $\Sigma_{1}^{h}$ have the same state dimensions and that the same happens for $\Sigma_{0}^{v}$ and $\Sigma_{1}^{v}$. Combining these realizations yields the following $(2,2)$-periodic 2D separable Roesser state space system $\Sigma_{p}^{2 D}$ :

$$
\begin{aligned}
& {\left[\begin{array}{l}
x^{h}(2 \ell+i+1,2 m+j) \\
x^{v}(2 \ell+i, 2 m+j+1)
\end{array}\right]} \\
& =\left[\begin{array}{cc}
A_{i}^{h} & 0 \\
A_{i j}^{v h} & A_{j}^{v}
\end{array}\right]\left[\begin{array}{l}
x^{h}(2 \ell+i, 2 m+j) \\
x^{v}(2 \ell+i, 2 m+j)
\end{array}\right] \\
& \quad+\left[\begin{array}{c}
B_{i}^{h} \\
B_{i j}^{v}
\end{array}\right] u(2 \ell+i, 2 m+j),
\end{aligned}
$$

$$
\begin{aligned}
w(2 \ell+i, 2 m+j) & \\
= & {\left[\begin{array}{ll}
C_{i j}^{h} & C_{j}^{v}
\end{array}\right]\left[\begin{array}{l}
x^{h}(2 \ell+i, 2 m+j) \\
x^{v}(2 \ell+i, 2 m+j)
\end{array}\right] } \\
& +D_{i j} u(2 \ell+i, 2 m+j),
\end{aligned}
$$

with $A_{i j}^{v h}=\tilde{B}_{j}^{v} \tilde{C}_{i}^{h}, B_{i j}^{v}=\tilde{B}_{j}^{v} D_{i}^{h}, C_{i j}^{h}=D_{j}^{v} \tilde{C}_{i}^{h}$, and $D_{i j}=D_{j}^{v} D_{i}^{h}$. 
Note that for each pair of fixed values of $i$ and $j$ this periodic 2D system is an invariant separable 2D state space system

$$
\Sigma_{(i, j)}=\left(A_{i}^{h}, A_{j}^{v}, A_{i j}^{v h}, B_{i}^{h}, B_{i j}^{V}, C_{i j}^{h}, C_{j}^{v}, D_{i j}\right) .
$$

Similar to what happens in the $1 \mathrm{D}$ case, we say that $\Sigma_{p}^{2 D}$ is obtained from $\Sigma_{(0,0)}, \Sigma_{(1,0)}, \Sigma_{(0,1)}$, and we $\Sigma_{(1,1)}$ and write $\Sigma_{p}^{2 D}=\left(\Sigma_{(0,0)}, \Sigma_{(1,0)}, \Sigma_{(0,1)}, \Sigma_{(1,1)}\right)$.

As shown in the following example, the 2D (2,2)-periodic Roesser state space system $\Sigma_{p}^{2 D}=\left(\Sigma_{(0,0)}, \Sigma_{(1,0)}, \Sigma_{(0,1)}, \Sigma_{(1,1)}\right)$ is not necessarily a realization of the $(2,2)$-periodic encoding map

$$
\Phi_{V^{0} H^{0}, V^{0} H^{1}, V^{1} H^{0}, V^{1} H^{1}} .
$$

Example 1. Consider the (2,2)-periodic encoding map

$$
\Phi_{V^{0} H^{0}, V^{0} H^{1}, V^{1} H^{0}, V^{1} H^{1}},
$$

with

$$
\begin{gathered}
H^{0}\left(z_{1}\right)=H_{0}^{0}+H_{1}^{0} z_{1}+H_{2}^{0} z_{1}^{2} \\
=\left[\begin{array}{ccc}
1+z_{1}^{2} & 1 & 0 \\
z_{1}^{2} & 1+z_{1} & 1 \\
1+z_{1} & 1 & 1 \\
1 & 1 & 1+z_{1}
\end{array}\right], \\
H^{1}\left(z_{1}\right)=H_{0}^{1}+H_{1}^{1} z_{1}+H_{2}^{1} z_{1}^{2} \\
=\left[\begin{array}{ccc}
1+z_{1} & 1 & 0 \\
1+z_{1}^{2} & 1+z_{1} & 1 \\
1 & 1+z_{1}^{2} & 1 \\
0 & 1 & 1
\end{array}\right], \\
V^{0}\left(z_{2}\right)=\left(1+z_{2}\right) I_{4} \quad V^{1}\left(z_{2}\right)=\left(1+2 z_{2}\right) I_{4} .
\end{gathered}
$$

Realizing $H^{0}\left(z_{1}\right)$ as in Proposition 1 we obtain the state-space realization $\Sigma_{0}^{h}=\left(A_{0}^{h}, B_{0}^{h}, \tilde{C}_{0}^{h}, D_{0}^{h}\right)$ with

$$
\begin{aligned}
& A_{0}^{h}=\left[\begin{array}{llll}
0 & 0 & 0 & 0 \\
1 & 0 & 0 & 0 \\
0 & 0 & 0 & 0 \\
0 & 0 & 0 & 0
\end{array}\right], \quad B_{0}^{h}=\left[\begin{array}{lll}
1 & 0 & 0 \\
0 & 0 & 0 \\
0 & 1 & 0 \\
0 & 0 & 1
\end{array}\right], \\
& \tilde{C}_{0}^{h}=\left[\begin{array}{llll}
0 & 1 & 0 & 0 \\
0 & 1 & 1 & 0 \\
1 & 0 & 0 & 0 \\
0 & 0 & 0 & 1
\end{array}\right], \quad D_{0}^{h}=\left[\begin{array}{lll}
1 & 1 & 0 \\
0 & 1 & 1 \\
1 & 1 & 1 \\
1 & 1 & 1
\end{array}\right] .
\end{aligned}
$$

Proceeding in the same way, we obtain a state-space realization $\Sigma_{1}^{h}=\left(A_{1}^{h}, B_{1}^{h}, \tilde{C}_{1}^{h}, D_{1}^{h}\right)$ for $H^{1}\left(z_{1}\right)$ with

$$
A_{1}^{h}=\left[\begin{array}{llll}
0 & 0 & 0 & 0 \\
1 & 0 & 0 & 0 \\
0 & 0 & 0 & 0 \\
0 & 0 & 1 & 0
\end{array}\right], \quad B_{1}^{h}=\left[\begin{array}{lll}
1 & 0 & 0 \\
0 & 0 & 0 \\
0 & 1 & 0 \\
0 & 0 & 0
\end{array}\right],
$$

$$
\tilde{C}_{1}^{h}=\left[\begin{array}{llll}
1 & 0 & 0 & 0 \\
0 & 1 & 1 & 0 \\
0 & 0 & 0 & 1 \\
0 & 0 & 0 & 0
\end{array}\right], \quad D_{1}^{h}=\left[\begin{array}{lll}
1 & 1 & 0 \\
1 & 1 & 1 \\
1 & 1 & 1 \\
0 & 1 & 1
\end{array}\right]
$$

As for $V^{0}\left(z_{2}\right)$ and $V^{1}\left(z_{2}\right)$, it is easily seen that they can be realized by $\Sigma_{0}^{v}=\left(A_{0}^{v}, \tilde{B}_{0}^{v}, C_{0}^{v}, D_{0}^{v}\right)$ and $\Sigma_{1}^{v}=$ $\left(A_{1}^{v}, \tilde{B}_{1}^{v}, C_{1}^{v}, D_{1}^{v}\right)$ with

$$
A_{0}^{v}=0_{4}, \quad \tilde{B}_{0}^{v}=C_{0}^{v}=D_{0}^{v}=I_{4}
$$

and

$$
A_{1}^{v}=0_{4}, \quad \tilde{B}_{0}^{v}=D_{0}^{v}=I_{4}, \quad C_{0}^{v}=2 I_{4},
$$

where $0_{4}$ denotes the $4 \times 4$ zero matrix.

For every $t_{2} \in \mathbb{N}_{0}$, consider

$$
\begin{array}{rlrl}
u\left(0, t_{2}\right) & =\left[\begin{array}{l}
0 \\
0 \\
1
\end{array}\right], & u\left(1, t_{2}\right) & =\left[\begin{array}{l}
0 \\
0 \\
0
\end{array}\right] \\
u\left(t_{1}, t_{2}\right) & =0, & t_{1} \geq 2 .
\end{array}
$$

From (5) it follows that, for $m \in \mathbb{N}_{0}, j=0,1$,

$$
\begin{aligned}
w(1,2 m+j) & =\left(V^{j}\left(z_{2}\right) H^{1}\left(z_{1}\right) u\left(z_{1}, z_{2}\right)\right)(1,2 m+j) \\
& =\left(V^{j}\left(z_{2}\right) \bar{u}\left(z_{1}, z_{2}\right)\right)(1,2 m+j),
\end{aligned}
$$

where

$$
\begin{aligned}
& \bar{u}(1,2 m+j) \\
& \quad=H_{0}^{1} u(1,2 m+j)+H_{1}^{1} u(0,2 m+j) \\
& \quad=\left[\begin{array}{lll}
1 & 1 & 0 \\
1 & 1 & 1 \\
1 & 1 & 1 \\
0 & 1 & 1
\end{array}\right]\left[\begin{array}{l}
0 \\
0 \\
0
\end{array}\right]+\left[\begin{array}{lll}
1 & 0 & 0 \\
0 & 1 & 0 \\
0 & 0 & 0 \\
0 & 0 & 0
\end{array}\right]\left[\begin{array}{l}
0 \\
0 \\
1
\end{array}\right]=\left[\begin{array}{l}
0 \\
0 \\
0 \\
0
\end{array}\right] .
\end{aligned}
$$

Thus

$$
w(1,2 m+j)=\left[\begin{array}{l}
0 \\
0 \\
0 \\
0
\end{array}\right], \quad m \in \mathbb{N}_{0}, \quad j=0,1
$$

or simply

$$
w\left(1, t_{2}\right)=\left[\begin{array}{l}
0 \\
0 \\
0 \\
0
\end{array}\right] \quad \text { for } t_{2} \in \mathbb{N}_{0} .
$$

On the other hand, using (11), we have

$$
w(1,0)=\left[\begin{array}{ll}
C_{10}^{h} & C_{0}^{v}
\end{array}\right]\left[\begin{array}{l}
x^{h}(1,0) \\
x^{v}(1,0)
\end{array}\right]+D_{10} u(1,0) .
$$


Note that, since the initial conditions must be zero (according to our definition of realization), $x^{v}(1,0)=0$ and $x^{h}(0,0)=0$. Moreover,

$$
\begin{aligned}
x^{h}(1,0) & =A_{0}^{h} x^{h}(0,0)+B_{0}^{h} u(0,0) \\
& =\left[\begin{array}{lll}
1 & 0 & 0 \\
0 & 0 & 0 \\
0 & 1 & 0 \\
0 & 0 & 1
\end{array}\right]\left[\begin{array}{l}
0 \\
0 \\
1
\end{array}\right]=\left[\begin{array}{l}
0 \\
0 \\
0 \\
1
\end{array}\right] .
\end{aligned}
$$

Hence

$$
\begin{aligned}
w(1,0) & =C_{10}^{h} x^{h}(1,0) \\
& =D_{0}^{v} \tilde{C}_{1}^{h} x^{h}(1,0) \\
& =I_{4}\left[\begin{array}{llll}
1 & 0 & 0 & 0 \\
0 & 1 & 1 & 0 \\
0 & 0 & 0 & 1 \\
0 & 0 & 0 & 0
\end{array}\right]\left[\begin{array}{l}
0 \\
0 \\
0 \\
1
\end{array}\right]=\left[\begin{array}{l}
0 \\
0 \\
1 \\
0
\end{array}\right],
\end{aligned}
$$

i.e., the output $w$ of the 2D (2,2)-periodic Roesser state space system $\Sigma_{p}^{2 D}$ corresponding to $v$ is different from the trajectory $w$ corresponding to $u$ according to (5).

However, the next theorem shows that it is possible to obtain periodic 2D separable Roesser model realizations for 2D periodic encoding maps (5) by independently realizing the operators $V^{0}, V^{1}$, and $H^{0}, H^{1}$, provided that $V^{0}$ and $V^{1}$ have the same column degrees and the same happens for $H^{0}$ and $H^{1}$.

Theorem 5. Consider the polynomial matrices $H^{0}\left(z_{1}\right), H^{1}\left(z_{1}\right) \in \mathbb{F}^{q \times k}\left[z_{1}\right]$, and assume that they have the same column degrees. Let $\Sigma_{i}^{h}$ be the realizations of $H^{i}\left(z_{1}\right), i=0,1$, obtained by Proposition 1$]$ Consider further the polynomial matrices $V^{0}\left(z_{2}\right), V^{1}\left(z_{2}\right) \in$ $\mathbb{F}^{q \times n}\left[z_{2}\right]$, and assume that they have the same column degrees. Let $\Sigma_{j}^{v}$ be the realizations of $V^{j}\left(z_{2}\right), j=0,1$, obtained by Proposition 1 Define the $2 D$ periodic Roesser separable model $\Sigma_{p}^{2 D}$ obtained from $\Sigma_{i}^{h}$ and $\Sigma_{j}^{v}$ as in (11). Then $\Sigma_{p}^{2 D}$ is a state space realization of the $2 D$ periodic encoding map given by (5).

Proof. Note that, since $H^{0}\left(z_{1}\right)$ and $H^{1}\left(z_{1}\right)$ have the same column degrees, it follows that the corresponding realizations $\Sigma_{i}^{h}=\left(A_{i}^{h}, B_{i}^{h}, \tilde{C}_{i}^{h}, D_{i}^{h}\right), i=0,1$, are such that $A_{0}^{h}=A_{1}^{h}$ and $B_{0}^{h}=B_{1}^{h}$. Let us consider $A^{h}:=$ $A_{0}^{h}=A_{1}^{h}$ and $B^{h}:=B_{0}^{h}=B_{1}^{h}$. For the same reason the realizations $\Sigma_{j}^{v}=\left(A_{j}^{v}, \tilde{B}_{j}^{v}, C_{j}^{v}, D_{j}^{v}\right), j=0,1$, are such that $A_{0}^{v}=A_{1}^{v}$ and $\tilde{B}_{0}^{v}=\tilde{B}_{1}^{v}$. Let $A^{v}:=A_{0}^{v}=A_{1}^{v}$ and $\tilde{B}^{v}:=\tilde{B}_{0}^{v}=\tilde{B}_{1}^{v}$.

Then, after simple, but cumbersome computations, one concludes that the output $w$ of $\Sigma_{p}^{2 D}=$ $\left(\Sigma_{(0,0)}, \Sigma_{(1,0)}, \Sigma_{(0,1)}, \Sigma_{(1,1)}\right)$, with

$$
\begin{gathered}
\Sigma_{(i, j)}=\left(A^{h}, A^{v}, A_{i j}^{v h}=\tilde{B}^{v} \tilde{C}_{i}^{h}, B^{h}, B_{i j}^{v}=\tilde{B}^{v} D_{i}^{h},\right. \\
\left.C_{i j}^{h}=D_{j}^{v} \tilde{C}_{i}^{h}, C_{j}^{v}, D_{i j}\right),
\end{gathered}
$$

which corresponds to the input $u$ and zero initial conditions $\left(x^{h}\left(0, t_{2}\right)=0, x^{v}\left(t_{1}, 0\right)=0\right)$, is such that, for $\ell, m \in \mathbb{N}_{0}, i, j=0,1$,

$$
\begin{aligned}
& w(2 \ell+i, 2 m+j) \\
& =D_{j}^{v} D_{i}^{h} v(2 \ell+i, 2 m+j) \\
& \quad+\sum_{t_{1} \geq 1} C_{i j}^{h}\left(A^{h}\right)^{t_{1}-1} B^{h} u\left(2 \ell+i-t_{1}, 2 m+j\right) \\
& \quad+\sum_{t_{2} \geq 1} C_{j}^{v}\left(A^{v}\right)^{t_{2}-1} B_{i j}^{v} v\left(2 \ell+i, 2 m+j-t_{2}\right) \\
& \quad+\sum_{t_{1}, t_{2} \geq 1} C_{j}^{v}\left(A^{v}\right)^{t_{2}-1} A_{i j}^{v h}\left(A^{h}\right)^{t_{1}-1} \\
& \quad \times B^{h} v\left(2 \ell+i-t_{1}, 2 m+j-t_{2}\right) .
\end{aligned}
$$

Let us now show that the codeword

$$
\tilde{w}=\left(V^{j}\left(z_{2}\right) H^{i}\left(z_{1}\right)\right) u
$$

equals $w$. For that purpose, note that, since $\Sigma_{0}^{h}=$ $\left(A^{h}, B^{h}, \tilde{C}_{0}^{h}, D_{0}^{h}\right)$ is a realization of $H^{0}$, we have that

$$
H^{0}\left(z_{1}\right)=D_{0}^{h}+\sum_{t_{1} \geq 1} \tilde{C}_{0}^{h}\left(A^{h}\right)^{t_{1}-1} B^{h} z_{1}^{t_{1}} .
$$

In the same way,

$$
\begin{aligned}
& H^{1}\left(z_{1}\right)=D_{1}^{h}+\sum_{t_{1} \geq 1} \tilde{C}_{1}^{h}\left(A^{h}\right)^{t_{1}-1} B^{h} z_{1}^{t_{1}}, \\
& V^{0}\left(z_{2}\right)=D_{0}^{v}+\sum_{t_{2} \geq 1} C_{0}^{v}\left(A^{v}\right)^{t_{2}-1} \tilde{B}^{v} z_{2}^{t_{2}} .
\end{aligned}
$$

and

$$
V^{1}\left(z_{2}\right)=D_{1}^{v}+\sum_{t_{2} \geq 1} C_{1}^{v}\left(A^{v}\right)^{t_{2}-1} \tilde{B}^{v} z_{2}^{t_{2}} .
$$

Thus

$$
\begin{aligned}
& \tilde{w}(2 \ell+i, 2 m+j) \\
& \quad=\sum_{\substack{0 \leq t_{1} \leq 2 k+i \\
0 \leq t_{2} \leq 2 l+j}} G(i, j) u\left(2 \ell+i-t_{1}, 2 m+j-t_{2}\right),
\end{aligned}
$$

where $G(i, j)$ is the coefficient of $z_{1}^{i} z_{2}^{j}$ of the polynomial matrix in $z_{1}$ and $z_{2}, V^{j}\left(z_{2}\right) H^{i}\left(z_{1}\right)$.

It is not difficult to check that

$$
\begin{aligned}
& V^{j}\left(z_{2}\right) H^{i}\left(z_{1}\right) \\
& =D_{j}^{v} D_{i}^{h}+\sum_{t_{1} \geq 1} D_{j}^{v} \tilde{C}_{i}^{h}\left(A^{h}\right)^{t_{1}-1} B^{h} z_{1}^{t_{1}} \\
& \quad+\sum_{t_{2} \geq 1} C_{j}^{v}\left(A^{v}\right)^{t_{2}-1} \tilde{B}^{v} D_{i}^{h} z_{2}^{t_{2}} \\
& \quad+\sum_{t_{1}, t_{2} \geq 1} C_{j}^{v}\left(A^{v}\right)^{t_{2}-1} \tilde{B}^{v} \tilde{C}_{i}^{h}\left(A^{h}\right)^{t_{1}-1} B^{h} z_{1}^{t_{1}} z_{2}^{t_{2}} .
\end{aligned}
$$


Taking into account that $C_{i j}^{h}=D_{j}^{v} \tilde{C}_{i}^{h}, B_{i j}^{v}=\tilde{B}^{v} D_{i}^{h}$ and $A_{i j}^{v h}=\tilde{B}^{v} \tilde{C}_{i}^{h}$, this allows us to conclude that $\tilde{w}=w$.

In order to study the minimality of the 2D state space realization 111 , we start by defining its lifted version.

4.3. Lifted 2D realization. Following the ideas of Aleixo and Rocha $(2017 ; 2018)$, consider the $(2,2)$-periodic 2D separable Roesser state space system $\Sigma_{p}^{2 D}$ given by (11) and define the lifted versions of the horizontal and vertical states as

$$
X^{h}(\ell, m)=\left[\begin{array}{c}
x^{h}(2 \ell, 2 m) \\
x^{h}(2 \ell, 2 m+1)
\end{array}\right]
$$

and

$$
X^{v}(\ell, m)=\left[\begin{array}{c}
x^{v}(2 \ell, 2 m) \\
x^{v}(2 \ell+1,2 m)
\end{array}\right],
$$

respectively; define also the lifted versions of the input and the output respectively as

$$
u^{L}(\ell, m)=\left[\begin{array}{c}
u(2 \ell, 2 m) \\
u(2 \ell+1,2 m) \\
u(2 \ell, 2 m+1) \\
u(2 \ell+1,2 m+1)
\end{array}\right]
$$

and

$$
w^{L}(\ell, m)=\left[\begin{array}{c}
w(2 \ell, 2 m) \\
w(2 \ell+1,2 m) \\
w(2 \ell, 2 m+1) \\
w(2 \ell+1,2 m+1)
\end{array}\right] .
$$

This yields the following 2D invariant separable Roesser model:

$$
\begin{array}{r}
{\left[\begin{array}{l}
X^{h}(\ell+1, m) \\
X^{v}(\ell, m+1)
\end{array}\right]=P\left[\begin{array}{l}
X^{h}(\ell, m) \\
X^{v}(\ell, m)
\end{array}\right]+Q u^{L}(\ell, m),} \\
w^{L}(\ell, m)=R\left[\begin{array}{l}
X^{h}(\ell, m) \\
X^{v}(\ell, m)
\end{array}\right]+S u^{L}(\ell, m),
\end{array}
$$

where the matrices $P, Q, R$ and $S$ are constant and can be decomposed as follows:

$$
P=\left[\begin{array}{cc}
P^{h} & 0 \\
P^{v h} & P^{v}
\end{array}\right], \quad Q=\left[\begin{array}{l}
Q^{h} \\
Q^{v}
\end{array}\right], \quad R=\left[\begin{array}{ll}
R^{h} & R^{v}
\end{array}\right],
$$

with the dimensions of the blocks determined by the dimensions of $X^{h}$ and $X^{v}$ and, moreover, where

$$
\begin{aligned}
& P^{h}=\left[\begin{array}{cc}
A_{1}^{h} A_{0}^{h} & 0 \\
0 & A_{1}^{h} A_{0}^{h}
\end{array}\right], \quad P^{v}=\left[\begin{array}{cc}
A_{1}^{v} A_{0}^{v} & 0 \\
0 & A_{1}^{v} A_{0}^{v}
\end{array}\right] \text {, } \\
& P^{v h}=\left[\begin{array}{cc}
A_{1}^{v} \tilde{B}_{0}^{v} \tilde{C}_{0}^{h} & \tilde{B}_{1}^{v} \tilde{C}_{0}^{h} \\
A_{1}^{v} \tilde{B}_{0}^{v} \tilde{C}_{1}^{h} A_{0}^{h} & \tilde{B}_{1}^{v} \tilde{C}_{1}^{h} A_{0}^{h}
\end{array}\right], \\
& Q^{h}=\left[\begin{array}{cccc}
A_{1}^{h} B_{0}^{h} & B_{1}^{h} & 0 & 0 \\
0 & 0 & A_{1}^{h} B_{0}^{h} & B_{1}^{h}
\end{array}\right], \\
& Q^{v}=\left[\begin{array}{cccc}
A_{1}^{v} \tilde{B}_{0}^{v} D_{0}^{h} & 0 & \tilde{B}_{1}^{v} D_{0}^{h} & 0 \\
A_{1}^{v} \tilde{B}_{0}^{v} \tilde{C}_{1}^{h} B_{0}^{h} & A_{1}^{v} \tilde{B}_{0}^{v} D_{1}^{h} & \tilde{B}_{1}^{v} \tilde{C}_{1}^{h} B_{0}^{h} & \tilde{B}_{1}^{v} D_{1}^{h}
\end{array}\right],
\end{aligned}
$$

$$
R^{h}=\left[\begin{array}{cc}
D_{0}^{v} \tilde{C}_{0}^{h} & 0 \\
D_{0}^{v} \tilde{C}_{1}^{h} A_{0}^{h} & 0 \\
C_{1}^{v} \tilde{B}_{0}^{v} \tilde{C}_{0}^{h} & D_{1}^{v} \tilde{C}_{0}^{h} \\
C_{1}^{v} \tilde{B}_{0}^{v} \tilde{C}_{1}^{h} A_{0}^{h} & D_{1}^{v} \tilde{C}_{1}^{h} A_{0}^{h}
\end{array}\right]
$$$$
R^{v}=\left[\begin{array}{cc}
C_{0}^{v} & 0 \\
0 & C_{0}^{v} \\
C_{1}^{v} A_{0}^{v} & 0 \\
0 & C_{1}^{v} A_{0}^{v}
\end{array}\right]
$$

$$
S=\left[\begin{array}{cccc}
D_{0}^{v} D_{0}^{h} & 0 & 0 & 0 \\
D_{0}^{v} \tilde{C}_{1}^{h} B_{0}^{h} & D_{0}^{v} D_{1}^{h} & 0 & 0 \\
C_{1}^{v} \tilde{B}_{0}^{v} D_{0}^{h} & 0 & D_{1}^{v} D_{0}^{h} & 0 \\
C_{1}^{v} \tilde{B}_{0}^{v} \tilde{C}_{1}^{h} B_{0}^{h} & C_{1}^{v} \tilde{B}_{0}^{v} D_{1}^{h} & D_{1}^{v} \tilde{C}_{1}^{h} B_{0}^{h} & D_{1}^{v} D_{1}^{h}
\end{array}\right] .
$$

We denote this 2D invariant lifted model by $\Sigma^{L}=(P, Q, R, S)$.

\section{Minimality}

Theorem 6. Let $H^{0}\left(z_{1}\right), H^{1}\left(z_{1}\right) \in \mathbb{F}^{q \times k}\left[z_{1}\right]$ be two column reduced encoders with the same column degrees, and let $V^{0}\left(z_{2}\right), V^{1}\left(z_{2}\right) \in \mathbb{F}^{q \times n}\left[z_{2}\right]$ be also two column reduced encoders with the same column degrees. Let further $\Sigma_{i}^{h}=\left(A^{h}, B^{h}, \tilde{C}_{i}^{h}, D_{i}^{h}\right)$ and $\Sigma_{j}^{v}=$ $\left(A^{v}, B^{v}, C_{j}^{v}, D_{j}^{v}\right)$ be respectively $1 D$ state space realizations of $H^{i}$ (of dimension $\delta_{h}$ ) and $V^{j}$ (of dimension $\delta_{v}$ ), $i, j=0,1$, obtained as in Proposition 11 Define the $2 D$ periodic Roesser separable model $\Sigma_{p}^{2 D}$ obtained from $\Sigma_{i}^{h}$ and $\Sigma_{j}^{v}$ as in (11). If the matrix

$$
M=\left[\begin{array}{ccc}
\tilde{C}_{0}^{h} & D_{0}^{h} & 0 \\
\tilde{C}_{1}^{h} A^{h} & \tilde{C}_{1}^{h} B^{h} & D_{1}^{h}
\end{array}\right]
$$




$$
\operatorname{rank}\left[\begin{array}{ll}
A^{h} B^{h} & B^{h}
\end{array}\right]\left|\left[\begin{array}{ll}
\left(A^{h}\right)^{3} B^{h} & \left(A^{h}\right)^{2} B^{h}
\end{array}\right]\right| \cdots \mid\left[\begin{array}{ll}
\left(A^{h}\right)^{4 \delta_{h}-1} B^{h} & \left(A^{h}\right)^{4 \delta_{h}-2} B^{h}
\end{array}\right]=\delta_{h}
$$

has full row rank and the matrix

$$
N=\left[\begin{array}{cc}
D_{0}^{v} & 0 \\
C_{1}^{v} B^{v} & D_{1}^{v} \\
A^{v} B^{v} & B^{v}
\end{array}\right]
$$

has full column rank, then $\Sigma_{p}^{2 D}$ is a minimal state space realization of the $2 D$ periodic encoding map $\Phi_{V^{0} H^{0}, V^{0} H^{1}, V^{1} H^{0}, V^{1} H^{1}}$ given by [5].

Proof. The proof of this theorem is a direct consequence of the next four lemmas.

The next lemma follows immediately from the definition of the lifted system.

Lemma 3. $\quad \Sigma_{p}^{2 D}$ is a minimal state space realization if and only if $\Sigma^{L}$ is minimal.

As a consequence of the previous lemma, to prove Theorem 6 we just need to prove that the lifted realization $\Sigma^{L}=(P, Q, R, S)$ given by (12) is separately locally controllable and separately locally observable. We start with the proof of the separate local controllability of $\Sigma^{L}$.

Lemma 4. In the conditions of Theorem 6 the realization $\Sigma^{L}=(P, Q, R, S)$ given by (12) is separately locally controllable.

Proof. By Lemma 11 we just have to prove that the matrices $\left(P^{h}, Q^{h}\right)$ and $\left(P^{v},\left[Q^{v} \mid P^{v h}\right]\right)$ are controllable, where the involved matrices are defined by (14) and (15) with $A_{1}^{h}=A_{0}^{h}=A^{h}, A_{1}^{v}=A_{0}^{v}=A^{v}$, $B_{1}^{h}=B_{0}^{h}=B^{h}$ and $B_{1}^{v}=\tilde{B}_{0}^{v}=\tilde{B}^{v}$.

By Proposition 2, we have that $\left(P^{h}, Q^{h}\right)$ is controllable if and only if

$$
\operatorname{rank}\left[Q^{h}\left|P^{h} Q^{h}\right| \cdots \mid\left(P^{h}\right)^{2 \delta_{h}-1} Q^{h}\right]=2 \delta_{h},
$$

which is equivalent to (18), and this last equality is true because $\left(A^{h}, B^{h}\right)$ is controllable by Proposition 1 (note that the matrix in the expression contains all the column blocks of the controllability matrix $\mathcal{C}^{h}$ of $\left.\left(A^{h}, B^{h}\right)\right)$.

In order to prove the controllability of $\left(P^{v},\left[Q^{v} \mid P^{v h}\right]\right)$, note that $P^{v}, P^{v h}$ and $Q^{v}$ are given by

$$
\begin{aligned}
& P^{v}=\left[\begin{array}{cc}
\left(A^{v}\right)^{2} & 0 \\
0 & \left(A^{v}\right)^{2}
\end{array}\right] \\
& P^{v h}=\left[\begin{array}{cc}
A^{v} B^{v} \tilde{C}_{0}^{h} & B^{v} \tilde{C}_{0}^{h} \\
A^{v} B^{v} \tilde{C}_{1}^{h} A^{h} & B^{v} \tilde{C}_{1}^{h} A^{h}
\end{array}\right] \\
& Q^{v}=\left[\begin{array}{cccc}
A^{v} B^{v} D_{0}^{h} & 0 & B^{v} D_{0}^{h} & 0 \\
A^{v} B^{v} \tilde{C}_{1}^{h} B^{h} & A^{v} B^{v} D_{1}^{h} & B^{v} \tilde{C}_{1}^{h} B^{h} & B^{v} D_{1}^{h}
\end{array}\right] .
\end{aligned}
$$

Applying block column permutations and defining the matrix

$$
M=\left[\begin{array}{ccc}
\tilde{C}_{0}^{h} & D_{0}^{h} & 0 \\
\tilde{C}_{1}^{h} A^{h} & \tilde{C}_{1}^{h} B^{h} & D_{1}^{h}
\end{array}\right],
$$

the pair $\left(P^{v},\left[Q^{v} \mid P^{v h}\right]\right)$ becomes

$$
\begin{aligned}
& \left(\left[\begin{array}{cc}
\left(A^{v}\right)^{2} & 0 \\
0 & \left(A^{v}\right)^{2}
\end{array}\right],\right. \\
& \left.\left[\left[\begin{array}{cc}
B^{v} & 0 \\
0 & B^{v}
\end{array}\right] M \mid\left[\begin{array}{cc}
A^{v} B^{v} & 0 \\
0 & A^{v} B^{v}
\end{array}\right] M\right]\right) .
\end{aligned}
$$

Therefore, by Proposition 2 we have that $\left(P^{v},\left[Q^{v} \mid P^{v h}\right]\right)$ is controllable if and only if (20), holds or, equivalently, (21) which is clearly true since the matrix $M$ has full row rank and $\left(A^{v}, B^{v}\right)$ is controllable by Proposition 1 .

To prove that the realization $\Sigma^{L}=(P, Q, R, S)$ given by (12) is separately locally observable, we will first prove the next auxiliary lemma.

Lemma 5. Let $\nu_{1}, \nu_{2}, \ldots, \nu_{k}$ be nonnegative integers and define the matrix $A=\operatorname{diag}\left(A_{1}, A_{2}, \ldots, A_{k}\right)$, where

$$
A_{i}=\left[\begin{array}{cccc}
0 & \cdots & \cdots & 0 \\
1 & & & \vdots \\
& \ddots & & \vdots \\
& & 1 & 0
\end{array}\right] \in \mathbb{F}^{\nu_{i} \times \nu_{i}}
$$

In the case where $\nu_{i}=0$ the $i$-th block of $A$ is void.

Consider two matrices $C_{0}, C_{1} \in \mathbb{F}^{n \times \bar{\nu}_{k}}$, with $\bar{\nu}_{j}=\sum_{i=1}^{j} \nu_{i}, j=1, \ldots, k$, such that $\left[C_{0}\right]_{\bar{\nu}_{1}, \bar{\nu}_{2}, \ldots, \bar{\nu}_{k}}$ and $\left[C_{1}\right]_{\bar{\nu}_{1}, \bar{\nu}_{2}, \ldots, \bar{\nu}_{k}}$ have full column rank, where $\left[C_{i}\right]_{\bar{\nu}_{1}, \bar{\nu}_{2}, \ldots, \bar{\nu}_{k}}$ represents the submatrix of $C_{i}, i=0,1$, with columns $\bar{\nu}_{1}, \bar{\nu}_{2}, \ldots, \bar{\nu}_{k}$.

\section{Then the matrix}

$$
\left[\begin{array}{l}
C_{0} \\
C_{1} A \\
C_{0} A^{2} \\
\vdots \\
C_{r} A^{\nu-1}
\end{array}\right]
$$

where $\nu=\max \nu_{i}, i=1, \ldots, k$ and $r=(\nu-1) \bmod 2$, has full column rank.

Proof. Define $\bar{\nu}_{j}^{(n)}=\bar{\nu}_{j}-n$ if $\bar{\nu}_{j}-n \geq 0$ (otherwise $\bar{\nu}_{j}^{(n)}$ is not defined). By assumption, $\left[C_{0}\right]_{\bar{\nu}_{1}, \bar{\nu}_{2}, \ldots, \bar{\nu}_{k}}$ has full column rank. Note that the columns $\bar{\nu}_{1}, \bar{\nu}_{2}, \ldots, \bar{\nu}_{k}$ of 


$$
\operatorname{rank}\left[\left[\begin{array}{cc}
B^{v} & 0 \\
0 & B^{v}
\end{array}\right] M\left[\begin{array}{cc}
A^{v} B^{v} & 0 \\
0 & A^{v} B^{v}
\end{array}\right] M\left|\left[\begin{array}{cc}
\left(A^{v}\right)^{2} B^{v} & 0 \\
0 & \left(A^{v}\right)^{2} B^{v}
\end{array}\right] M\left[\begin{array}{cc}
\left(A^{v}\right)^{3} B^{v} & 0 \\
0 & \left(A^{v}\right)^{3} B^{v}
\end{array}\right] M\right| \ldots\right]=2 \delta_{v} \quad(20)
$$

$$
\left.\operatorname{rank}\left[\left[\begin{array}{cc}
B^{v} & 0 \\
0 & B^{v}
\end{array}\right]\left[\begin{array}{cc}
A^{v} B^{v} & 0 \\
0 & A^{v} B^{v}
\end{array}\right]\left[\begin{array}{cc}
\left(A^{v}\right)^{2} B^{v} & 0 \\
0 & \left(A^{v}\right)^{2} B^{v}
\end{array}\right]\left[\begin{array}{cc}
\left(A^{v}\right)^{3} B^{v} & 0 \\
0 & \left(A^{v}\right)^{3} B^{v}
\end{array}\right] \quad \cdots\right] \operatorname{diag}(M)\right]
$$

$A$ are zero, which implies that the columns of the same index of

$$
\left[\begin{array}{c}
C_{1} A \\
C_{0} A^{2} \\
C_{1} A^{3} \\
\vdots
\end{array}\right]
$$

are also zero. Moreover,

$$
\left[C_{1} A\right]_{\bar{\nu}_{1}^{(1)}, \bar{\nu}_{2}^{(1)}, \ldots, \bar{\nu}_{k}^{(1)}}=\left[C_{1}\right]_{\bar{\nu}_{1}, \bar{\nu}_{2}, \ldots, \bar{\nu}_{k}}
$$

has full column rank.

In the same way, the columns $\bar{\nu}_{1}, \bar{\nu}_{1}^{(1)} \bar{\nu}_{2}, \bar{\nu}_{2}^{(1)} \ldots, \bar{\nu}_{k}, \bar{\nu}_{k}^{(1)}$ of $A^{2}$ are also zero and therefore the columns of the same index of

$$
\left[\begin{array}{l}
C_{0} A^{2} \\
C_{1} A^{3} \\
\vdots
\end{array}\right]
$$

are also zero, and $\left[C_{0} A^{2}\right]_{\bar{\nu}_{1}^{(2)}, \bar{\nu}_{2}^{(2)}, \ldots, \bar{\nu}_{k}^{(2)}}=\left[C_{0}\right]_{\bar{\nu}_{1}, \bar{\nu}_{2}, \ldots, \bar{\nu}_{k}}$ has full column rank.

Proceeding analogously, it is easy to check that

$$
\left[\begin{array}{l}
C_{0} \\
C_{1} A \\
C_{0} A^{2} \\
\vdots \\
C_{r} A^{\nu-1}
\end{array}\right]
$$

has full column rank.

Lemma 6. In the conditions of Theorem 6 the realization $\Sigma^{L}=(P, Q, R, S)$ given by (12) is separately locally observable.

Proof. By Lemma 2, it is sufficient to prove that the matrices $\left(P^{v}, R^{v}\right)$ and $\left(P^{h},\left[\begin{array}{c}R^{h} \\ P^{v h}\end{array}\right]\right)$ are observable, where the involved matrices are defined by (14) and (16) with $A_{1}^{h}=A_{0}^{h}=A^{h}, A_{1}^{v}=A_{0}^{v}=A^{v}, B_{1}^{h}=B_{0}^{h}=B^{h}$ and $B_{1}^{v}=\tilde{B}_{0}^{v}=\tilde{B}^{v}$.

The observability of $\left(P^{v}, R^{v}\right)$ follows immediately by Proposition 2 and Lemma 5

In order to prove the observability of $\left(P^{h},\left[\begin{array}{c}R^{h} \\ P^{v h}\end{array}\right]\right)$ note that this pair is equal to

$$
\left(\left[\begin{array}{cc}
\left(A^{h}\right)^{2} & 0 \\
0 & \left(A^{h}\right)^{2}
\end{array}\right],\left[\begin{array}{cc}
D_{0}^{v} \tilde{C}_{0}^{h} & 0 \\
D_{0}^{v} \tilde{C}_{1}^{h} A^{h} & 0 \\
C_{1}^{v} B^{v} \tilde{C}_{0}^{h} & D_{1}^{v} \tilde{C}_{0}^{h} \\
C_{1}^{v} B^{v} \tilde{C}_{1}^{h} A^{h} & D_{1}^{v} \tilde{C}_{1}^{h} A^{h} \\
\hline A^{v} B^{v} \tilde{C}_{0}^{h} & B^{v} \tilde{C}_{0}^{h} \\
A^{v} B^{v} \tilde{C}_{1}^{h} A^{h} & B^{v} \tilde{C}_{1}^{h} A^{h}
\end{array}\right]\right) \text {. }
$$

Applying block row permutations and defining the matrix

$$
N=\left[\begin{array}{cc}
D_{0}^{v} & 0 \\
C_{1}^{v} B^{v} & D_{1}^{v} \\
A^{v} B^{v} & B^{v}
\end{array}\right]
$$

that has full column rank, by assumption, the pair (22) can be written as

$$
\left(\left[\begin{array}{cc}
\left(A^{h}\right)^{2} & 0 \\
0 & \left(A^{h}\right)^{2}
\end{array}\right],\left[\frac{N\left[\begin{array}{cc}
\tilde{C}_{0}^{h} & 0 \\
0 & \tilde{C}_{0}^{h}
\end{array}\right]}{N\left[\begin{array}{cc}
\tilde{C}_{1}^{h} A^{h} & 0 \\
0 & \tilde{C}_{1}^{h} A^{h}
\end{array}\right]}\right]\right) .
$$

The rest of the proof is analogous to the final part of the proof of Lemma4taking in account Lemma 5 .

\section{Acknowledgment}

This research was supported by the Portuguese National Funding Agency for Science, Research and Technology (FCT), within the Center for Research 
and Development in Mathematics and Applications (CIDMA) (project UID/MAT/04106/2019). It was also partially supported by the project POCI-01 -0145-FEDER-006933-SYSTEC-Research Center for Systems and Technologies, funded by FEDER funds through COMPETE2020_Programa Operacional Competitividade e Internacionalização (POCI), and with national funds through FCT-Fundação para a Ciência e a Tecnologia.

\section{References}

Aleixo, J.C. and Rocha, P. (2017). Roesser model representation of 2D periodic behaviors: The (2,2)-periodic SISO case, 10th International Workshop on Multidimensional $(n D)$ Systems (nDS), Zielona Góra, Poland, pp. 1-6.

Aleixo, J.C. and Rocha, P. (2018). On the state space realization of separable periodic 2D systems, 2018 European Control Conference, Limassol, Cyprus, pp. 2300-2305.

Aleixo, J.C., Rocha, P. and Willems, J.C. (2011). State space representation of SISO periodic behaviors, 2011 50th IEEE Conference on Decision and Control/European Control Conference, Orlando, FL, USA, pp. 1545-1550.

Basu, S. and Swamy, M.N.S. (2002). Editorial preface to special issue on multidimensional signals and systems, IEEE Transactions on Circuits and Systems I: Fundamental Theory and Applications 49(6): 709-714.

Charoenlarpnopparut, C. and Bose, N. (2001). Grobner bases for problem solving in multidimensional systems, Multidimensional Systems and Signal Processing 12(3): 365-376.

Climent, J.-J., Herranz, V., Perea, C. and Tomás, V. (2009). A systems theory approach to periodically time-varying convolutional codes by means of their invariant equivalent, in M. Bras-Amorós and T. Høholdt (Eds), Applied Algebra, Algebraic Algorithms and Error-Correcting Codes, Springer, Berlin/Heidelberg, pp. 73-82.

Costello, D.J. (1974). Free distance bounds for convolutional codes, IEEE Transactions on Information Theory 20(3): 356-365.

Felstrom, A.J. and Zigangirov, K.S. (1999). Time-varying periodic convolutional codes with low-density parity-check matrix, IEEE Transactions on Information Theory 45(6): 2181-2191.

Fornasini, E., Pinho, T., Pinto, R. and Rocha, P. (2015). Minimal realizations of syndrome formers of a special class of 2D codes, in R. Pinto et al. (Eds), Coding Theory and Applications, Springer International Publishing, Cham, pp. 185-193.

Fornasini, E. and Pinto, R. (2004). Matrix fraction descriptions in convolutional coding, Linear Algebra and Its Applications 392(C): 119-158.

Fornasini, E. and Valcher, M. (1994). Algebraic aspects of two-dimensional convolutional codes, IEEE Transactions on Information Theory 40(4): 1068-1082.
Fornasini, E. and Valcher, M. (1998). Multidimensional systems with finite support behaviors: Signal structure, generation and detection, SIAM Journal on Control and Optimization 36(2): 760-779.

Galkowski，K. (1996). The Fornasini-Marchesini and the Roesser models: Algebraic methods for recasting, IEEE Transactions on Automatic Control 41(1): 107-112.

Galkowski, K. (2001). State-Space Realisations of Linear 2$D$ Systems with Extensions to the General $n D(n>2)$ Case, Vol. 263, Lecture Notes in Control and Information Sciences, Springer, London.

Gluesing-Luersen, H., Rosenthal, J. and Weiner, P. (2000). Duality between mutidimensinal convolutional codes and systems, in F. Colonius et al. (Eds), Advances in Mathematical Systems Theory: A Volume in Honor of Diedrich Hinrichsen, Birkhauser, Boston, MA, pp. 135-150.

Gluesing-Luerssen, H. and Schneider, G. (2007). State space realizations and monomial equivalence for convolutional codes, Linear Algebra and Its Applications 425(2): 518-533.

Guardia, G.G.L. (2019). Asymptotically good convolutional codes, Linear and Multilinear Algebra 67(7): 1483-1494.

Hinamoto, T. (1980). Realizations of a state-space model from two-dimensional input-output map, IEEE Transactions on Circuits and Systems 27(1): 36-44.

Jangisarakul, P. and Charoenlarpnopparut, C. (2011). Algebraic decoder of multidimensional convolutional code: Constructive algorithms for determining syndrome decoder and decoder matrix based on Grobner basis, Multidimensional Systems and Signal Processing 22(1): 67-81.

Kaczorek, T. (2001). Elimination of finite eigenvalues of the 2D Roesser model by state feedbacks, International Journal of Applied Mathematics and Computer Science 11(2): 369-376.

Kailath, T. (1980). Linear Systems, Prentice-Hall, Englewood Cliffs, NJ.

Kuijper, M. and Polderman, J. (2004). Reed-Solomon list decoding from a system theoretic perspective, IEEE Transactions on Information Theory IT-50(2): 259-271.

Kuijper, M. and Willems, J.C. (1997). A behavioral framework for periodically time varying systems, Proceedings of the 36th IEEE Conference on Decision and Control, San Diego, CA, USA, Vol. 3, pp. 2013-2016.

Lobo, R., Bitzer, D.L. and Vouk, M.A. (2012). On locally invertible encoders and multidimensional convolutional codes, IEEE Transactions on Information Theory 58(3): 1774-1782.

Mooser, M. (1983). Some periodic convolutional codes better than any fixed code (corresp.), IEEE Transactions on Information Theory 29(5): 750-751.

Napp, D., Perea, C. and Pinto, R. (2010). Input-state-output representations and constructions of finite support 2D convolutional codes, Advances in Mathematics of Communications 4(4): 533-545. 
Napp, D., Pereira, R., Pinto, R. and Rocha, P. (2019). Periodic state-space representations of periodic convolutional codes, Cryptography and Communications 11(4): $585-595$.

Palazzo, R. (1993). A time-varying convolutional encoder better than the best time-invariant encoder, IEEE Transactions on Information Theory 39(3): 1109-1110.

Pinho, T. (2016). Minimal State-Space Realizations of $2 D$ Convolutional Codes, $\mathrm{PhD}$ thesis, University of Aveiro, Aveiro.

Pinho, T., Pinto, R. and Rocha, P. (2014). Realization of $2 \mathrm{D}$ convolutional codes of rate $1 / \mathrm{n}$ by separable Roesser models, Designs, Codes and Cryptography 70(1): 241-250.

Rosenthal, J. (2001). Connections between linear systems and convolutional codes, in B. Marcus and J. Rosenthal (Eds), Codes, Systems and Graphical Models, Springer-Verlag, New York, NY, pp. 39-66.

Rosenthal, J. and York, E.V. (1999). BCH convolutional codes, IEEE Transactions on Automatic Control 45(6): 1833-1844.

Valcher, M. and Fornasini, E. (1994). On 2D finite support convolutional codes: An algebraic approach, Multidimensional Systems and Signal Processing 5(3): 231-243.

Weiner, P. (1998). Multidimensional Convolutional Codes, PhD dissertation, University of Notre Dame, South Bend, IN.

Zerz, E. (2000). Topics in Multidimensional Linear Systems Theory, Lecture Notes in Control and Information Sciences, Vol. 256, Springer-Verlag, London.
Diego Napp received his $\mathrm{PhD}$ degree in mathematics from the University of Groningen, The Netherlands, in 2008. From 2008 to 2018 he enjoyed several fellowships and research positions in Portugal and Spain. He is currently an assistant professor at the Department of Mathematics of the University of Alicante, Spain. His research interests are in systems and coding theory, algebra and cryptography.

Ricardo Pereira graduated in applied mathematics from the University of Aveiro in 2001, where he obtained his PhD degree in mathematics in 2006. Since then he has been an assistant professor at the Department of Mathematics of the University of Aveiro. His interests are mainly in the behavioral approach to systems and control and convolutional coding.

Raquel Pinto graduated in applied mathematics/computer science from the University of Porto in 1991 and received her $\mathrm{PhD}$ degree in mathematics from the University of Aveiro in 2003. She is an assistant professor at the Department of Mathematics of the University of Aveiro. Her research interests cover the behavioral approach to systems theory and convolutional coding.

Paula Rocha graduated in applied mathematics from the University of Porto (PT) in 1983, and obtained her $\mathrm{PhD}$ in systems and control in 1990 from the University of Groningen (NL). She has held positions at TU Delft (NL) and at the University of Aveiro (PT), and is currently a full professor at the Department of Electrical and Computer Engineering, FEUP (PT). Her main interests are in the behavioral approach to systems and control, the connection between systems and codes, multidimensional (nD) systems, and control of biomedical systems.

Received: 29 November 2018

Revised: 25 February 2019

Accepted: 31 March 2019 